\title{
Reheating as a Surface Effect
}

\author{
Kari Enqvist, ${ }^{1,2}$ Shinta Kasuya, ${ }^{2}$ and Anupam Mazumdar ${ }^{3}$ \\ ${ }^{1}$ Department of Physical Sciences, P.O. Box 64, FIN-00014, University of Helsinki, Finland \\ ${ }^{2}$ Helsinki Institute of Physics, P.O. Box 64, FIN-00014, University of Helsinki, Finland \\ ${ }^{3}$ The Abdus Salam International Centre for Theoretical Physics, I-34100, Trieste, Italy
}

(Received 29 April 2002; published 7 August 2002)

\begin{abstract}
We describe a new mechanism for reheating the Universe through evaporation of a surface charge of a fragmented inflaton condensate. We show that for a range of Yukawa coupling of the inflaton to the matter sector evaporation gives rise to a much smaller reheat temperature compared to the standard perturbative decay. As a consequence, reheating through a surface effect could solve the gravitino and moduli overproduction problem in inflationary models without fine tuning the Yukawa sector.
\end{abstract}

PACS numbers: $98.80 . \mathrm{Cq}$

Inflation gives naturally the initial condition for the hot big bang cosmology by reheating the Universe through a conversion of the energy density stored in the inflaton field into radiation of elementary particles [1]. However, as it is well known, the entropy thus dumped into the Universe may pose a problem for big bang nucleosynthesis. This constraints the number densities of light quanta (or massive quanta which later decay) at the time of reheating, which is given by [2] $n / s \approx \eta\left(T_{R} / 10^{11}\right) \mathrm{GeV}$, where $n$ is number density, $s$ is the entropy density of the thermal bath with a reheat temperature $T_{R}$, and $\eta \approx 10^{-11}$ is the matterto-entropy ratio at the time of big bang nucleosynthesis. For a successful big bang nucleosynthesis one requires $n / s \leq 10^{-13}$, which translates into $T_{R} \leq 10^{9} \mathrm{GeV}$ [3]. Such a low reheat temperature is a challenge to inflation models as well as a perennial problem. For example, in models involving supersymmetry and superstrings one is often faced with an overproduction of gravitinos and moduli unless the reheat temperature can somehow be made low $[2,3]$.

Reheating is based on the decay of the inflaton field while it oscillates about its minimum [4]. The amplitude of the oscillations gradually decreases as the Universe expands, and the energy density redshifts as $\rho \propto a^{-3}$, where $a$ is the scale factor of the Universe. There exist two conceptually different realizations for the decay: direct (and slow) decay of the inflaton quanta, and a resonant conversion of the inflaton field to decay products by a process called preheating [5].

The decay lifetime of the inflaton can be computed in the limit $m \gg H$, where $m$ is the inflaton mass and $H$ is the expansion of the Universe. In particular, decay of an inflaton to a pair of fermions with an interaction $\mathcal{L}_{\text {int }}=$ $h \phi \bar{\psi} \psi$, when $m \gg m_{\psi}$, is given by

$$
\Gamma(\phi \rightarrow \bar{\psi} \psi)=\frac{h^{2} m}{8 \pi},
$$

where $h$ is the Yukawa coupling smaller than 1. In order to estimate the reheat temperature one equates the decaying inflaton energy density to the radiation energy density; $\rho_{r}=\left(\pi^{2} / 30\right) g_{*} T_{R}^{4}$, at the moment when $\Gamma=H$. Assuming that thermalization occurs quickly, one obtains a reheat temperature

$$
T_{R}=\left(\frac{90}{8 \pi^{3} g_{*}}\right)^{1 / 4} \quad \sqrt{\Gamma M_{P}} \sim 0.1 \sqrt{\Gamma M_{P}},
$$

where $g_{*} \sim \mathcal{O}(100)$ is the relativistic degrees of freedom at the time of reheating, and $M_{P}=1.2 \times 10^{19} \mathrm{GeV}$.

Preheating requires specific conditions which may or may not occur. However, the important point is that the standard reheating phenomena is a volume effect. If instead of the inflaton decaying inside some fixed volume it were to evaporate through the surface, the decay rate would naturally be suppressed by the ratio

$$
\frac{\text { area }}{\text { volume }} \propto R^{-1},
$$

where $R$ is the effective size of an object whose surface is evaporating. The larger the radius, the smaller is the evaporation rate, and therefore, the smaller is the reheat temperature. Reheating through a surface effect would thus be a natural way to achieve low reheat temperatures.

Note that such a phenomenon takes place in a selfgravitating system such as a black hole. Indeed, it has been claimed that in the case of a self-gravitating object there holds a holographic principle according to which all the degrees of freedom lie on the surface [6]. A black hole with a mass $M_{b h}$ evaporates from its gravitational radius $r_{g}=2 M_{b h} / M_{P}^{2}$ as a black body with temperature inversely proportional to the radius of a black hole $T_{b h}=\left(M_{\mathrm{P}}^{2} / 8 \pi M_{b h}\right)$ [7]. However, because of the small density contrast in the early Universe implied by the anisotropies of the cosmic microwave background, a sufficient number of primordial black holes might be very difficult to form.

However, a nontopological defect, which might encompass an inflaton condensate within a subhorizon radius, would typically also decay through its surface. An example 
is the $Q$ ball [8] that can exist in theories with scalar fields carrying a conserved global $U(1)$ charge. The $Q$ balls are long lived nontopological solitonic ground-state solutions for a fixed charge $Q$. This means that the energy of a $Q$-ball configuration is less than a collection of free scalars carrying an equivalent charge. The $Q$-ball field configuration is given by $\phi(x, t)=e^{i \omega t} \varphi(x)$, its energy and charge are given by

$$
\begin{aligned}
& E=\int d^{3} x\left[\frac{1}{2} \omega^{2} \varphi^{2}+\frac{1}{2}\left|\nabla \phi^{2}\right|+V(\phi)\right], \\
& Q=\omega \int \varphi^{2} d^{3} x .
\end{aligned}
$$

The potential $V(\phi)$ has a global minimum at the origin and it is invariant under global $U(1)$ transformation. Depending on the slope of the potential it is possible to have energetically stable $Q$ ball, for which we require $E \leq \mu Q$, where $\mu$ is given by

$$
\mu=\min \left[\sqrt{\frac{2 V(\phi)}{\phi^{2}}}\right] .
$$

In order to form $Q$ balls a negative pressure is required for the scalar condensate to fragment $[9,10]$. Several analytical and numerical studies have verified this in the context of the minimal supersymmetric standard model flat direction Affleck-Dine condensate [11].

An example of a potential which can lead to a $Q$-ball formation is given by

$$
V(\varphi)=m^{2}\left(1+|K| \log \left[\frac{\varphi^{2}}{M_{\mathrm{P}}^{2}}\right]\right) \varphi^{2} .
$$

The one-loop logarithmic running of $\varphi$ appears due to the presence of finite Yukawa couplings to bosons and fermions, or, if $\varphi$ has some gauge interactions. Such a correction to the potential appears in supersymmetry and in particular, if $\varphi$ has gauge interactions then $K<0$ [10]. In nonsupersymmetric theories it is possible to obtain $K<0$, provided the Yukawa coupling to the fermions dominate, or if there are more fermionic loops than bosonic ones.

Let us now assume that our inflaton sector has a global $U(1)$ symmetry with a running mass governed by Eq. (6). Although the inflaton is often taken to be a real, there seems to be no reason why it should not be a complex field (and perhaps this would be even more natural). Then chaotic inflation occurs for initial values $\varphi>M_{P}$ [1]. The running mass inflation has been a topic of recent investigation and interesting details can be found in [12].

We note that right after the end of inflation, when the inflaton condensate starts to oscillate at $H \approx m$, the condensate can fragment due to the perturbations in $\phi$ field and eventually form a $Q$ ball. In this case $Q$ balls can form due to the oscillations in $\phi$ field $[11,13]$.

The oscillating inflaton field will fragment into $Q$ balls if $|K|<0$. To see this, the potential Eq. (6) can be ex- panded in a form $V(\varphi) \propto \varphi^{2+2|K|}$. The equation of state for such a potential is given by $p=-(K / 2) \rho$ [14]. Hence, oscillating inflaton field does not behave effectively as pressureless matter, as it is usually assumed, but feels a negative pressure. A perturbation in the inflaton field will then obey [10]

$$
\delta \ddot{\varphi}_{k}=-\frac{K k^{2}}{2} \delta \varphi_{k} .
$$

Therefore, the fluctuation of the field grows on scale $k=$ $2 \pi / \lambda$ in time

$$
\delta \varphi_{k}=\delta \varphi_{i k} \exp \left(\sqrt{\frac{|K| k^{2}}{2} t}\right),
$$

where $t=0$ corresponds to the beginning of coherent oscillations of the inflaton field, and $\delta \phi_{i}$ is the initial perturbation in the field when $H \sim m$. Here, obviously, we have neglected the effect of expansion, but we keep in mind that a physical momentum redshifts during the expansion of the Universe, and as long as the wavelength of the excited mode is well within the Hubble length it is possible to follow the fragmentation of the inflaton condensate. Note that perturbations of wavelength $\lambda$ take a finite time to grow nonlinear $t \sim(1 / 2 \pi)(2 /|K|)^{1 / 2} \lambda$. This may be taken as an estimate for the time it takes to fragment the inflaton condensate and to form lumps of charged $Q$ balls [11].

A potential of the form Eq. (6) generically gives rise to a thick-wall $Q$ ball with a Gaussian profile $\varphi(r) \propto$ $\exp \left(-|K| m^{2} r^{2} / 2\right)$. The size of such a $Q$ ball can be estimated by [10]

$$
R \equiv|K|^{-1 / 2} m^{-1} .
$$

Therefore, a $Q$ ball of size $R$ forms when

$$
H_{f} \propto \frac{1}{t} \sim \mathcal{O}(1)|K| m .
$$

The exact proportionality depends on details which we are neglecting at the moment for an order of magnitude estimate.

The total charge accumulated by a $Q$ ball will therefore depend on $Q=2 \omega \varphi_{0}^{2} V=(8 \pi / 3) m \varphi_{0}^{2} R^{3}$, where $\varphi_{0}$ is the inflaton field value at which the $Q$ ball forms. Since $|K|<1$, we can approximate the decay in the amplitude of the oscillations by $\varphi_{0} \sim \varphi_{i}\left(H_{f} / H_{i}\right)$ similar to a matter dominated era, where $\varphi_{i} \sim M_{\mathrm{P}}$, denotes the end of inflation in chaotic model, and $H_{i} \sim m$ when the oscillations begin. Therefore, the total charge of a $Q$ ball can be given by

$$
Q \sim \frac{3}{8 \pi}\left(\frac{1}{|K|^{1 / 2} m}\right)^{3}|K|^{2} m M_{\mathrm{P}}^{2}=\frac{3}{8 \pi}|K|^{1 / 2}\left(\frac{M_{P}}{m}\right)^{2} .
$$

Note, that the charge is fixed by the ratio of $m / M_{P}$ determined by the anisotropies seen in the cosmic micro wave 
background radiation [12]

$$
\frac{\Delta \rho}{\rho} \sim \frac{m}{M_{P}} \sim 10^{-5},
$$

which results in $m \sim 10^{13} \mathrm{GeV}$.

The evaporation of a $Q$ ball through the decay of a charged massless fermion has been studied in $[15,16]$. Analytical estimates in the thin-wall case have yielded an upper limit on the evaporation rate, which is saturated even in thick-wall case for $h \varphi_{0} \geq \omega$, where $h$ is the Yukawa coupling [15]. The evaporation rate is bounded by

$$
\frac{d Q}{d t} \leq \frac{\omega^{3} A}{192 \pi^{2}}
$$

where in our case $\omega=m$. The above estimation is valid for strong Yukawa coupling. In Eq. (13), $A=4 \pi R^{2}$ denote the surface area of a $Q$ ball, and the above expression simplifies to

$$
\frac{d Q}{d t} \leq \frac{1}{48 \pi} \frac{m}{|K|}
$$

Therefore, we can estimate the decay rate of a $Q$ ball in the process of reheating the Universe

$$
\Gamma=\frac{1}{Q} \frac{d Q}{d t} \approx \frac{m}{18|K|^{3 / 2}}\left(\frac{m}{M_{\mathrm{P}}}\right)^{2} .
$$

In general $K$ and $h$ are not independent quantities but are related to each other by $K \sim C h^{2} / 16 \pi^{2}$, where $C$ is effective number of bosonic and fermionic loops. Even though we are in the strong coupling limit, the decay rate mimics that of a Planck suppressed interaction of the inflaton made $Q$ ball to the matter fields. The estimated reheat temperature is then given by

$$
T_{R} \leq \frac{1}{|K|^{3 / 4}} 10^{8} \mathrm{GeV} \approx 5 C^{-3 / 4} h^{-3 / 2} \times 10^{9} \mathrm{GeV} .
$$

If the inflaton sector does not belong to a hidden sector then it is very natural that inflaton coupling to other matter fields is sufficiently large, i.e., $1 \gtrsim h \gtrsim\left(m / M_{P}\right)$. As an example, we may consider $C \sim 10$, and $h \sim 1$, we obtain $|K| \sim 0.1$. The resulting reheat temperature is then $T_{R} \approx 5 \times$ $10^{8} \mathrm{GeV}$. We notice that the dependence of the reheat temperature on $|K|$ is in general rather weak. For such a low reheat temperature gravitinos and moduli can never be over produced from a thermal bath [2]. Therefore, reheating through a surface of a $Q$ ball solves the gravitino and moduli problem without invoking very small Yukawa coupling. This is the main application of reheating through a surface.

We notice that for couplings $1 \gtrsim h \gtrsim \mathcal{O}\left(m / M_{P}\right)$, Eq. (16) predicts in this particular model much lower reheat temperature than direct decay of inflaton, which from Eq. (1), yields

$$
T_{R} \sim 2 \times 10^{14} h \mathrm{GeV} .
$$

In the weak Yukawa coupling case when $h \varphi_{0} \ll m$ (so that $h \ll m / M_{\mathrm{P}}$ ), the evaporation rate of a $Q$ ball [15], and subsequently the decay rate are bounded by

$$
\frac{d Q}{d t} \leq \frac{1}{16} \frac{h \varphi_{0}}{|K|}, \quad \Gamma \leq \frac{h \pi}{6|K|^{1 / 2}} \frac{m^{2}}{M_{P}},
$$

and the limit on the reheat temperature reads now

$$
T_{R} \leq 0.1\left(\frac{h}{|K|^{1 / 2}}\right)^{1 / 2} m \sim 0.3 C^{-1 / 4} m .
$$

Note that the dependence on $h$ is canceled out. Thus for a sufficiently small $h \ll\left(m / M_{P}\right) \sim 10^{-5}$ the reheat temperature saturates and would be larger than through the perturbative decay of inflaton. This result may appear counterintuitive, as it suggests that the evaporation rate is faster than the perturbative decay rate for small $h$. The point is that a fermion gets an effective mass $m_{\psi} \sim h \varphi_{0}$ inside a $Q$ ball. The corresponding Compton wavelength $m_{\psi}^{-1}$ then actually becomes larger than the size of a $Q$ ball for very small $h$. In fact the ratio between the evaporation and perturbative decay rate goes as $\sim m_{\psi}^{-1} / R>1$, and therefore the evaporation through a surface effect given by Eq. (18) cannot be trusted any longer. This is an example where a simple relationship given by Eq. (3) does not hold.

We have pointed out a new possibility of reheating the Universe through an evaporation of a surface. We have shown that without invoking very low fine tuned Yukawa coupling, the evaporation of a surface charge from the inflaton condensate inevitably gives much smaller reheat temperature compared to the standard perturbative decay of inflaton. This new mechanism could be perhaps the simplest option to solve the gravitino, polonyi, and dilaton problems in general [3].

Even though we have considered $Q$-ball formation and reheating of the Universe in a chaotic inflationary scenario, it is conceivable that similar behavior could arise in many other inflaton models with an effective $U(1)$ global charge. It is quite possible that the inflaton condensate fragmentation and $Q$-ball formation can occur even if $U(1)$ would be slightly broken. Hence, in addition to the standard reheating, which is a volume effect, for a range of parameters the Universe may be reheated in a novel way, through surface evaporation of a $Q$ ball.

A.M. is thankful to Rouzbeh Allahverdi and Altug Ozpineci for helpful discussion, and S.K. is thankful to M. Kawasaki for a useful discussion. A. M. acknowledges the support of The Early Universe Network HPRN-CT2000-00152, and a kind hospitality of the Helsinki Institute of Physics where this work has been carried out. 
[1] For a review, see A.D. Linde, Particle Physics and Inflationary Cosmology (Harwood, Chur, Switzerland, 1990).

[2] J. Ellis, J.E. Kim, and D. V. Nanopoulos, Phys. Lett. B 145, 181 (1984); J. Ellis, D. V. Nanopoulos, K. A. Olive, and S-J. Rey, Astropart. Phys. 4, 371 (1996).

[3] See for a review, S. Sarkar, Rep. Prog. Phys. 59, 1493 (1996).

[4] E. W. Kolb and M.S. Turner, The Early Universe (Addison-Wesley, Reading, MA, 1990).

[5] J. Traschen and R. Brandenberger, Phys. Rev. D 42, 2491 (1990); L. A. Kofman, A. D. Linde, and A. A. Starobinsky, Phys. Rev. Lett. 73, 3195 (1994).

[6] G.'t Hooft, gr-qc/9310026.

[7] S. W. Hawking, Commun. Math. Phys. 43, 199 (1975).

[8] S. Coleman, Nucl. Phys. B262, 263 (1985); T. D. Lee and Y. Pang, Phys. Rep. 221, 251 (1982).
[9] A. Kusenko and M. Shaposhnikov, Phys. Lett. B 418, 46 (1998).

[10] K. Enqvist and J. McDonald, Nucl. Phys. B538, 321 (1999).

[11] S. Kasuya and M. Kawasaki, Phys. Rev. D 61, 041301 (2000); 62, 023512 (2000); K. Enqvist, A. Jokinen, T. Multamäki, and I. Vilja, Phys. Rev. D 63, 083501 (2001).

[12] A. R. Liddle and D. H. Lyth, Cosmological Inflation and Large-Scale Structure (Cambridge University Press, Cambridge, United Kingdom, 2000).

[13] S. Kasuya, Phys. Lett. B 515, 121 (2001).

[14] M. S. Turner, Phys. Rev. D 28, 1243 (1983).

[15] A. Cohen, S. Coleman, H. Georgi, and A. Manohar, Nucl. Phys. B272, 301 (1986).

[16] T. Multamäki and I. Vilja, Nucl. Phys. B574, 130 (2000). 